\title{
Diffuse Alveolar Hemorrhage: An Uncommon Manifestation of Vaping-associated Lung Injury
}

\author{
Rodger Wilhite ${ }^{1}$, Tarang Patel ${ }^{2}$, Ethan Karle ${ }^{2}$, Shyam Shankar ${ }^{1}$, Armin Krvavac $^{3}$ \\ 1. Pulmonary, Critical Care and Environmental Medicine, University of Missouri Health Care, Columbia, USA 2. \\ Internal Medicine, University of Missouri Health Care, Columbia, USA 3. Pulmonary and Critical Care Medicine, \\ University of Missouri Health Care, Columbia, USA
}

Corresponding author: Armin Krvavac, krvavaca@health.missouri.edu

\begin{abstract}
Vaping involves the use of a device to deliver aerosolized nicotine and tetrahydrocannabinol (THC) oils to the lungs. Vaping continues to increase in popularity; however, because it is a novel drug delivery system there is little evidence regarding its safety and long-term consequences. Here, we present a 22 -year-old Caucasian male who was admitted with acute hypoxic respiratory failure and massive hemoptysis. Contrasted computed tomography of the chest demonstrated ground glass opacities throughout all lung fields and bilateral pulmonary emboli. Bronchoalveolar lavage revealed increased red blood cell counts in serial aliquots, consistent with the diagnosis of diffuse alveolar hemorrhage (DAH). An extensive workup did not reveal an etiology for the DAH. However, further history was obtained, and the patient divulged daily vaping of THC. E-cigarette, or vaping, product use associated lung injury (EVALI) consists of a myriad of different lung injury patterns. Our case illustrates an uncommon presentation of EVALI with DAH and multiple pulmonary emboli.
\end{abstract}

Received 12/16/2019

Review began 12/26/2019 Review ended 12/29/2019 Published 12/31/2019

๑) Copyright 2019

Wilhite et al. This is an open access article distributed under the terms of the Creative Commons Attribution License CC-BY 3.0., which permits unrestricted use, distribution, and reproduction in any medium, provided the original author and source are credited.
Categories: Pulmonology, Public Health, Environmental Health

Keywords: inhalational lung injury, diffuse alveolar hemorrhage, pulmonary medicine, electronic cigarette, tetrahydrocannabinol, pulmonary embolism, evali, pneumonitis, vaping-induced lung injury, vaping-associated lung injury

\section{Introduction}

Electronic cigarette (e-cigarette) use or vaping involves the use of a device to heat liquids in order to deliver an aerosolized product to the lungs. E-cigarette, or vaping, product use associated lung injury (EVALI) has reached epidemic proportions with 1,888 reported cases and 37 deaths related to vaping [1]. EVALI has become a public health crisis threatening our nation's youth due to marketing directed towards adolescents and easy modification of devices that allows the introduction of tetrahydrocannabinol (THC) oils [2,3]. The diagnosis of EVALI is difficult to elucidate as a myriad of radiographic patterns including acute eosinophilic pneumonia, organizing pneumonia, lipoid pneumonia, diffuse alveolar damage, hypersensitivity pneumonitis, and diffuse alveolar hemorrhage (DAH) have been described [4]. We illustrate a patient with EVALI that presents with DAH and pulmonary embolism.

\section{Case Presentation}

A 22-year-old Caucasian male with no significant medical history was admitted for acute hypoxemic respiratory failure and massive hemoptysis. Physical examination revealed an afebrile and tachycardic young male in severe respiratory distress, oxygen saturating of $89 \%$ on 15 liters per minute of supplemental oxygen via a non-rebreather mask. He exhibited use of accessory respiratory muscles and diffuse rhonchi bilaterally. He underwent emergent endotracheal intubation in the setting of massive hemoptysis. Laboratory testing revealed neutrophil predominant (83.9\%) leukocytosis of $27.82 \times 10^{9} / \mathrm{L}$, hemoglobin of $12.7 \mathrm{~g} / \mathrm{dL}$, and thrombocytopenia of $129 \times 10^{9} / \mathrm{L}$. Electrolytes, renal function, liver enzymes, and coagulation studies were all within normal limits. Procalcitonin, N-terminal pro B-type natriuretic peptide, and C-reactive protein were elevated at $0.92 \mathrm{ng} / \mathrm{mL}, 634 \mathrm{pg} / \mathrm{mL}$, and $16.04 \mathrm{mg} / \mathrm{dL}$ respectively. Lactic acid was normal at 1.5 $\mathrm{mmol} / \mathrm{L}$. The urine drug screen was positive for cannabinoids.

A computed tomography (CT) scan of the chest was obtained and demonstrated diffuse ground-glass opacities and multiple pulmonary emboli (Figures 1,2). 


\section{Cureus}

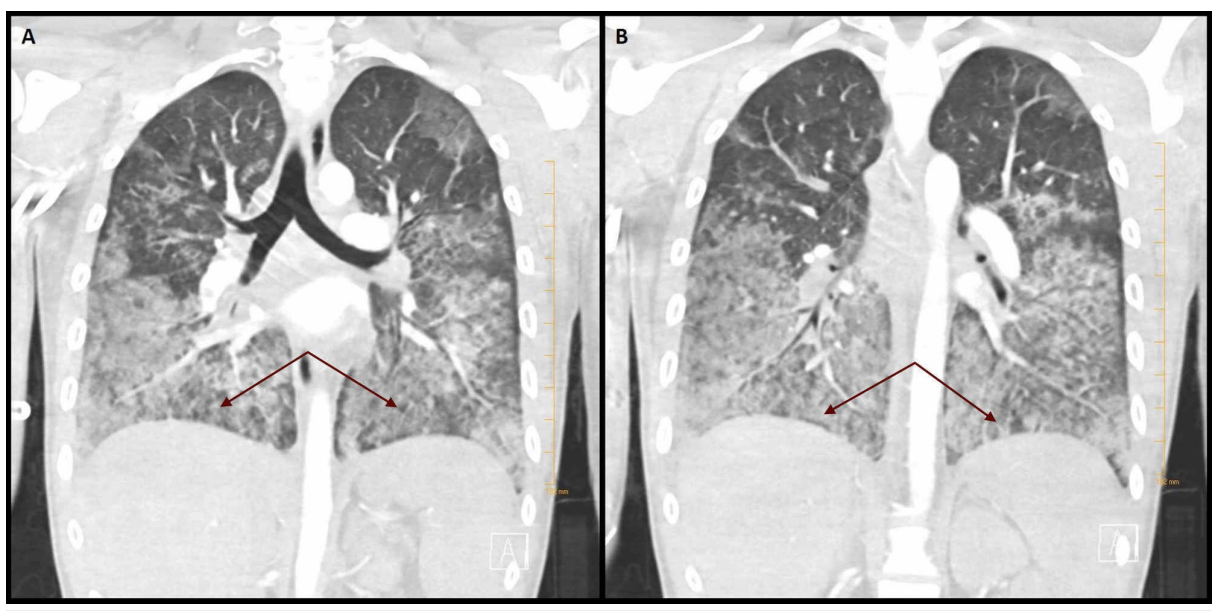

FIGURE 1: Computed tomography of the chest.

(A, B) Coronal section of computed tomography of the chest (arrows) demonstrating bilateral lower lobe predominant ground-glass opacities.

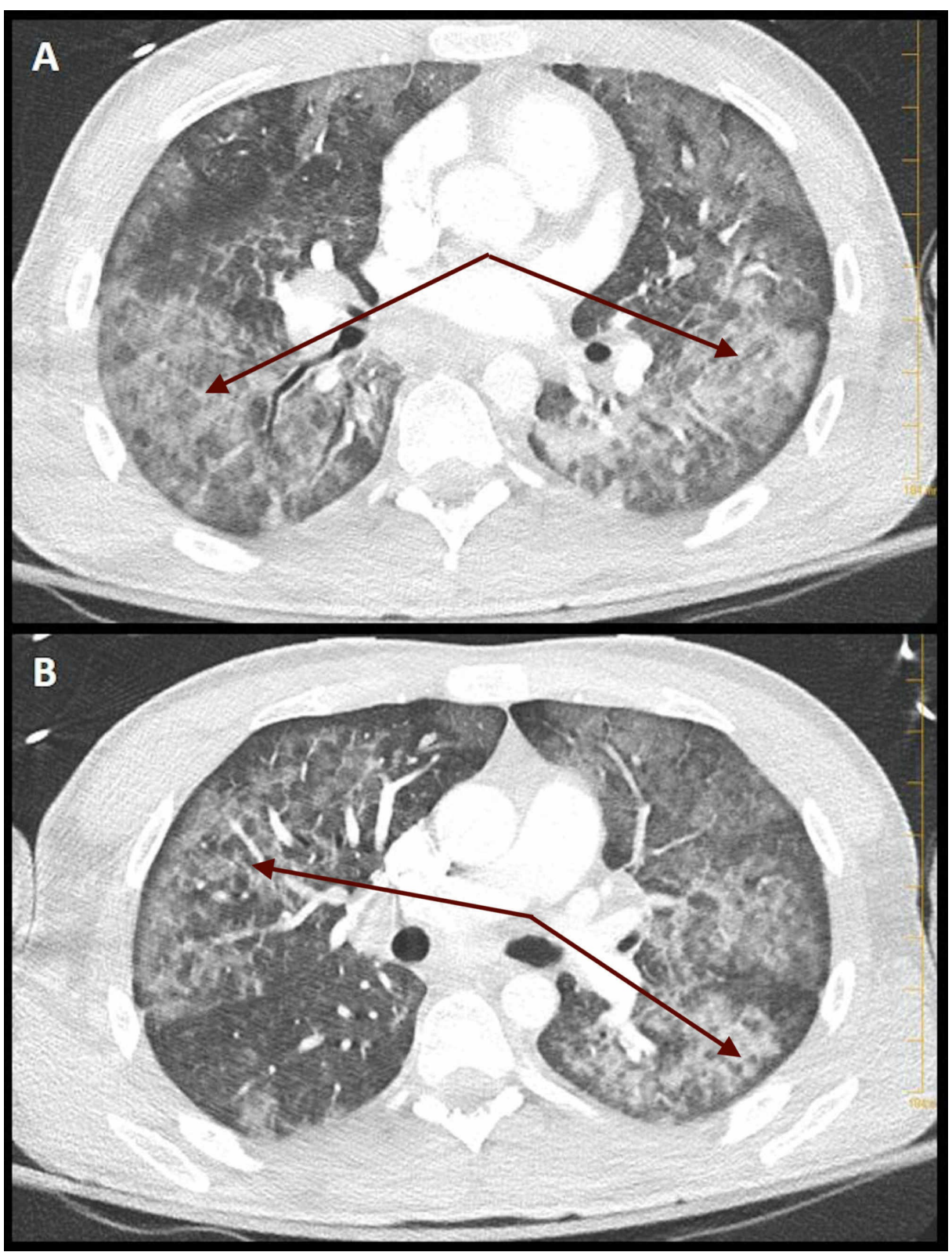




\section{Cureus}

FIGURE 2: Computed tomography of the chest.

(A, B) Axial section of computed tomography of the chest (arrows) demonstrating bilateral ground-glass opacities.

The patient underwent bronchoscopy and bronchoalveolar lavage, which revealed increasing red blood cells in three serial aliquots, consistent with DAH. Further investigation to explicate the etiology for DAH was unremarkable, and workup for autoimmune diseases, infectious causes, hematologic, and oncologic causes was negative (Tables 1-4).

$\begin{array}{lc}\text { Test } & \text { Result } \\ \text { Anti-Ro } & <4.9 \text { RLU } \\ \text { Anti-La } & <3.3 \text { RLU } \\ \text { Scl-70 } & <1.2 \text { RLU } \\ \text { Anti-Jo-1 } & <2.2 \text { RLU } \\ \text { Anti-ds-DNA } & \text { Negative } \\ \text { ANA } & \text { Negative } \\ \text { Anti-PR3 } & <2.3 \text { RLU } \\ \text { Anti-MPO } & <3.2 \text { RLU } \\ \text { Anti-GBM lgG } & <2.9 \text { RLU } \\ \text { ACE level } & 12 \text { U/L }\end{array}$

\section{TABLE 1: Autoimmune Diagnostic Evaluation of Diffuse Alveolar Hemorrhage}

Anti-ds-DNA, double stranded-deoxyribonucleic acid; ANA, anti-nuclear antibody; anti-PR3, anti-proteinase 3 antibody; anti-MPO, antimyeloperoxidase antibody; anti-GBM, anti-glomerular basement membrane antibody; ACE, angiotensin-converting enzyme. 


\section{Cureus}

\begin{tabular}{|l|l|}
\hline Test & Result \\
\hline RPP & Non-reactive \\
Sputum cultures & Negative fungal, bacterial, AFB \\
Blood cultures & Negative fungal, bacterial, AFB \\
Urine streptococcal antigen & Negative \\
Urine legionella antigen & Negative \\
Urine histoplasma antigen & Negative \\
HIV & Non-reactive \\
Hantavirus antibody & Negative \\
Anaplasma IgG & $<1: 64$ \\
CMV DNA & Not detected \\
Lyme disease titers IgG, IgM & Negative \\
RMSF titers IgG, IgM & $<1: 64$ \\
Ehrlichia PCR & Not detected \\
Fungal BAL antigen & Negative \\
\hline
\end{tabular}

\section{TABLE 2: Infectious Diagnostic Evaluation of Diffuse Alveolar Hemorrhage}

RPP, respiratory pathogens panel; HIV, human immunodeficiency virus; CMV, cytomegalovirus; RMSF, Rocky Mountain spotted fever; PCR, polymerase chain reaction; BAL, bronchoalveolar lavage; AFB, acid-fast bacilli.

\begin{tabular}{|l|l|}
\hline \hline Test & Result \\
\hline Peripheral blood smear & Negative for malignant cells \\
Lymph node FNA & Negative for malignant cells \\
Flow cytometry & Negative for malignant cells \\
Cytology BAL & Negative for malignant cells \\
\hline TABLE 3: Neoplastic Diagnostic Evaluation of Diffuse Alveolar Hemorrhage \\
\hline FNA, fine-needle aspiration; BAL, bronchoalveolar lavage.
\end{tabular}




\title{
Cureus
}

\begin{tabular}{|l|l|}
\hline Test & Result \\
\hline Factor V Leiden mutation analysis & Mutation not detected \\
\hline PT $20210 G$ A mutation & Mutation not detected \\
Anti-cardiolipin antibody & $<1$ RLU \\
Protein C & $81 \%$ \\
Protein S & $91 \%$ \\
Lupus anticoagulant antibody & Not detected \\
\hline
\end{tabular}

\section{TABLE 4: Hematologic Diagnostic Evaluation of Diffuse Alveolar Hemorrhage}

\author{
PT, prothrombin.
}

The patient subsequently divulged routine vaping with THC containing products that were purchased off the streets. His clinical condition improved after initiation of methylprednisolone with eventually transition to an oral prednisone taper.

\section{Discussion}

The recent recognition of EVALI is perplexing since vaping products were introduced in 2009. A major driver of the recent epidemic may be the increasing popularity of vaping among adolescents. Monitoring the future surveyed more than 40,000 adolescents and uncovered that the prevalence of vaping among them increased by more than twofold over the past two years [5]. Another contributor to the recent rise in cases may be the introduction of THC oils into vaping products. In the largest published case series of EVALI, $80 \%$ of the 53 patients reported vaping of THC oils [3]. Lastly, the variation in clinical symptoms and radiographic findings among various cases may have contributed to a lack of recognition prior to the recent epidemic, as case reports of e-cigarette-induced lung injury can be traced all the way to 2012 in the literature [6].

EVALI is reported most commonly among younger male patients, with presenting symptoms of fever, shortness of breath, and cough. Nearly all cases present with bilateral opacities on chest imaging, a neutrophil predominant leukocytosis $>11,000 / \mathrm{mm}^{3}$, and an elevated erythrocyte sedimentation rate. Clinicians should consider EVALI as the cause of respiratory failure in adolescent patients who endorse a history of vaping and present with bilateral radiographic opacities on chest imaging [3]. Although most cases present with bilateral opacities, the reported patterns on CT scans of the chest are much more heterogeneous [7].

The variety of radiographic presentations suggests that the pathophysiology of EVALI may be multifactorial. EVALI has been associated with the accumulation of lipid-laden macrophages (LLMs), although it is unclear if LLMs represent a pathophysiologic cause of lung injury [8,9]. Additionally, vaping appears to be directly toxic via thermal injury as e-cigarette aerosols can reach temperatures extreme enough to damage the respiratory epithelium [10]. Analyses of urine samples of e-cigarette users have demonstrated elevated levels of heavy metals [11]. Lastly, propylene glycol and glycerol produced by vaping have been associated with increased distal airway injury [12]. Limited case reports have implicated heavy metals, propylene glycol, and glycerol in the propagation of DAH [13-15]. Furthermore, our patient presents with a pulmonary embolism. Although it is unclear if there is a relationship between vaping and the development of pulmonary embolism, we know that there is a clear association between tobacco use and hypercoagulability [16].

\section{Conclusions}

At this time, the causative agent or agents and pathologic mechanisms of EVALI remain poorly understood. Our case exhibits an uncommon presentation of EVALI with DAH and multiple pulmonary emboli. It is unclear if there is a relationship between vaping and the development of pulmonary embolism. However, there is a clear association between tobacco use and hypercoagulability. Clinicians should continue to remain vigilant for new cases of EVALI given the various possible clinical, radiographic, and pathologic manifestations.

\section{Additional Information \\ Disclosures}

Human subjects: Consent was obtained by all participants in this study. Conflicts of interest: In 
compliance with the ICMJE uniform disclosure form, all authors declare the following: Payment/services info: All authors have declared that no financial support was received from any organization for the submitted work. Financial relationships: All authors have declared that they have no financial relationships at present or within the previous three years with any organizations that might have an interest in the submitted work. Other relationships: All authors have declared that there are no other relationships or activities that could appear to have influenced the submitted work.

\section{References}

1. Siegel DA, Jatlaoui TC, Koumans EH, et al.: Update: interim guidance for health care providers evaluating and caring for patients with suspected e-cigarette, or vaping, product use associated lung injury-United States, October. MMWR Morb Mortal Wkly Rep. 2019, 68:919-927. 10.15585/mmwr.mm6841e3

2. Gentzke AS, Creamer M, Cullen KA, et al.: Vital signs: tobacco product use among middle and high school students-United States, 2011-2018. Morbidity and Mortality Weekly. MMWR Morb Mortal Wkly Rep. 2019, 68:157-164. 10.15585/mmwr.mm6806e1

3. Layden JE, Ghinai I, Pray I, et al.: Pulmonary illness related to e-cigarette use in Illinois and Wisconsinpreliminary report. N Engl J Med. 2019, 10.1056/NEJMoa1911614

4. Christiani DC: Vaping-induced lung injury. N Engl J Med. 2019, 10.1056/NEJMe1912032

5. Miech R, Johnston L, O'Malley PM, Bachman JG, Patrick ME: Trends in adolescent vaping, 2017-2019. N Engl J Med. 2019, 381:1490-1491. 10.1056/NEJMc1910739

6. McCauley L, Markin C, Hosmer D: An unexpected consequence of electronic cigarette use . Chest. 2012, 141:1110-1113. 10.1378/chest.11-1334

7. Henry TS, Kanne JP, Kligerman SJ: Imaging of vaping-associated lung disease . N Engl J Med. 2019, 381:14861487. 10.1056/NEJMc1911995

8. Betancourt SL, Martinez-Jimenez S, Rossi SE, Truong MT, Carrillo J, Erasmus JJ: Lipoid pneumonia: spectrum of clinical and radiologic manifestations. AJR Am J Roentgenol. 2010, 194:103-109. 10.2214/AJR.09.3040

9. Romero F, Shah D, Duong M, et al.: A pneumocyte-macrophage paracrine lipid axis drives the lung toward fibrosis. Am J Respir Cell Mol Biol. 2015, 53:74-86. 10.1165/rcmb.2014-03430C

10. Mukhopadhyay S, Mehrad M, Dammert P, et al.: Lung biopsy findings in severe pulmonary illness associated with e-cigarette use (vaping). Am J Clin Pathol. 2020, 153:30-39. 10.1093/ajcp/aqz182

11. Goniewicz ML, Smith DM, Edwards KC, et al.: Comparison of nicotine and toxicant exposure in users of electronic cigarettes and combustible cigarettes. JAMA Netw Open. 2018, 1:e185937. 10.1001/jamanetworkopen.2018.5937

12. Chaumont $\mathrm{M}$, van de Borne $\mathrm{P}$, Bernard $\mathrm{A}$, et al.: Fourth generation e-cigarette vaping induces transient lung inflammation and gas exchange disturbances: results from two randomized clinical trials. Am J Physiol Lung Cell Mol Physiol. 2019, 316:L705-L719. 10.1152/ajplung.00492.2018

13. Agustin M, Yamamoto M, Cabrera F, Eusebio R: Diffuse alveolar hemorrhage induced by vaping. Case Rep Pulmonol. 2018, 2018:9724530. 10.1155/2018/9724530

14. Bakre SA, Al-Farra TS, Al-Farra S: Diffuse alveolar damage and e-cigarettes: case report and review of literature. Respir Med Case Rep. 2019, 28:100935. 10.1016/j.rmcr.2019.100935

15. Long JL, Devabhaktuni S, Hadique S, Jain P: Diffuse alveolar hemorrhage due to electronic cigarette use . Am Thorac Soc. 2016, 193:A1862.

16. Cheng YJ, Liu ZH, Yao FJ, et al.: Current and former smoking and risk for venous thromboembolism: a systematic review and meta-analysis. PLoS Med. 2013, 10:1001515. 10.1371/journal.pmed.1001515 